\title{
Developing a Programmable STEM Detector for the Scanning Electron Microscope
}

Benjamin W. Caplins ${ }^{1}$, Jason D. Holm ${ }^{1}$ and Robert R. Keller ${ }^{1}$

1. Applied Chemicals and Materials Division, National Institute of Standards and Technology, Boulder, CO, United States.

The scanning electron microscope (SEM) is traditionally used to analyze bulk samples, relying on signals generated by secondary and reflected/back-scattered electrons and photons to generate images. For relatively thin samples, forward-scattered electrons contain a wealth of information derived from the scattering volume. Diffraction within crystalline samples generates Bragg and Kikuchi scattered electrons which are sensitive to the crystallography and orientation of the material in addition to the presence of crystal defects. Scattering within amorphous samples leads to incoherent scattering relating to the mass thickness and/or atomic number of the material while coherent scattering can be related to the pairwise radial distribution function of the constituent atoms. Due to the reduced scattering volume of transmitted electrons relative to reflected electrons, the information derived from transmission measurements is generally obtained at a resolution equal-to or better-than bulk techniques. Scanning transmission electron microscopy (STEM) in the SEM (STEM-in-SEM) a promising technique to characterize nanoscale systems without the equipment overhead of a dedicated STEM.

One difficulty in making practical use of the forward-scattered electrons lies in the limitations of current STEM detectors. Broadly, STEM detectors can be categorized as either imaging detectors or integrating detectors. Imaging detectors give the user direct access to the sample's diffraction pattern at each beam position, but are generally slow ( $\sim 1000$ frames/second) and require (and permit) extensive off-line data analysis. On the other hand, integrating detectors can operate at high speeds ( $\mathrm{kHz}$ to $\mathrm{MHz})$ with excellent signal-to-noise, but cannot easily determine what features in the diffraction pattern are being measured, making image interpretation potentially difficult or even impossible in some situations.

Herein, we share progress made in our laboratory towards developing a new type of STEM detector that offers a hybrid approach. The basic detector design is shown in Figure 1a (see Ref. [1] for details). Transmitted electrons strike a scintillator screen placed directly beneath the sample, which serves to convert the electrons into photons. These photons are first imaged to a digital micromirror device (DMD) and then reimaged to a CMOS camera and/or an integrating photodetector (PMT). The DMD is a two-dimensional array $(1024 \times 768)$ of individually addressable mirrors which can be rapidly programmed to tilt up or down (towards the CMOS camera or PMT).

This programmable STEM (p-STEM) detector is currently used in two distinct modes. In 'diffraction' mode, all of the mirrors are tilted towards the CMOS camera giving direct access to the full diffraction pattern from a sample (Figure 1b). In 'imaging' mode, a user-defined subset of the mirrors is tilted towards the integrating photodetector which is synchronized with the microscope scan generator to create a real-space image. The DMD allows the user to easily switch between these modes during imaging sessions. A careful determination of the mapping functions between the scintillator, DMD, and CMOS camera allows the user to relate each mirror on the DMD to a scattering direction (Figure 1c).

Proof-of-principle data on a range of samples show diffraction contrast. In particular, we highlight the ability to generate contrast based on the crystallographic orientation of graphene. A dark-field image 
based on integrating reflections of a particular graphene grain shows contrast completely absent in the secondary-electron and bright-field image of the same area (Figure 2a). A color-composite of several different dark-field images from a large field-of-view is shown in Figure $2 b$.

The presented data show that the p-STEM detector is a promising new detector design for STEM-inSEM. Directly inspecting the diffraction pattern from a sample allows the user to orient their sample and select the appropriate electron beam parameters. The ability to quantitively define an arbitrary region of the diffraction pattern to integrate allows the user to specify dark-field conditions beyond simple circular or annular masks.

[1] B Jacobson et al, Proceedings of SPIE (2015) p. 93760K.

[2] This work is a contribution of the US Government and is not subject to United States copyright.
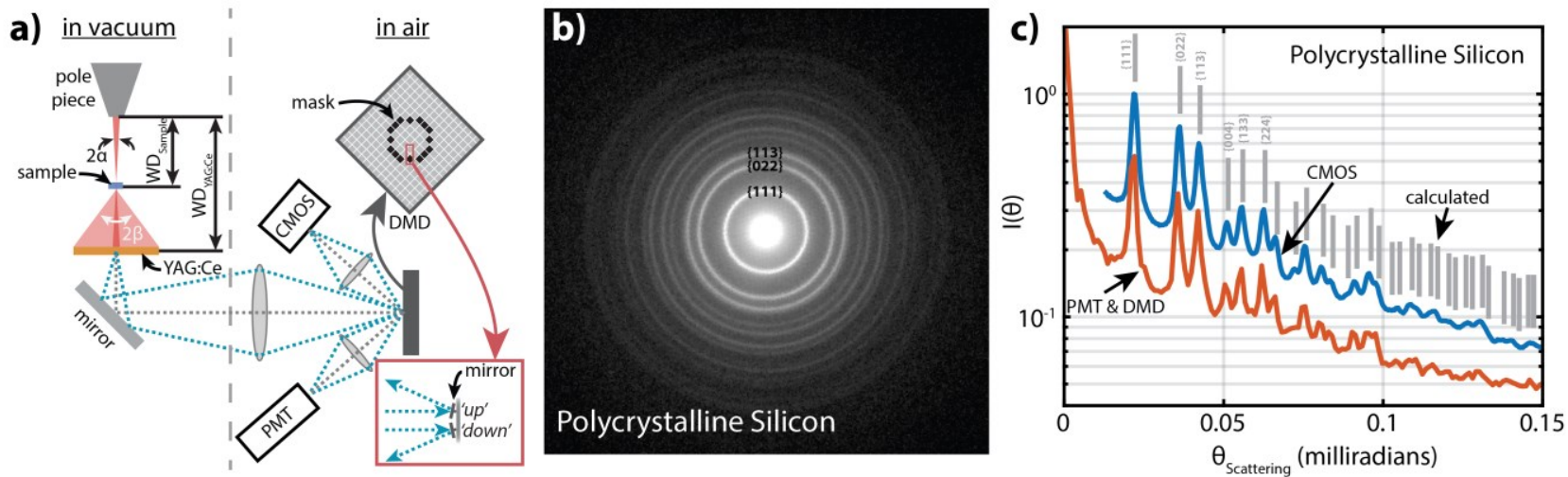

Figure 1. a) Electron detector schematic. The forward-scattered electrons are converted to photons on a scintillator screen which are optically imaged first to a DMD and then to a CMOS camera and/or integrating photodetector (PMT). b) A diffraction pattern from a polycrystalline silicon sample collected with all the DMD mirrors tilted towards the CMOS camera. c) Vertical bars: Calculated diffraction peak locations for silicon. Top curve: The annular integration of a polycrystalline silicon diffraction pattern collected on the CMOS camera. Bottom curve: Scattering data collected on the same sample by measuring the output of an integrating photodetector while programming a series of thin annular masks onto the DMD.
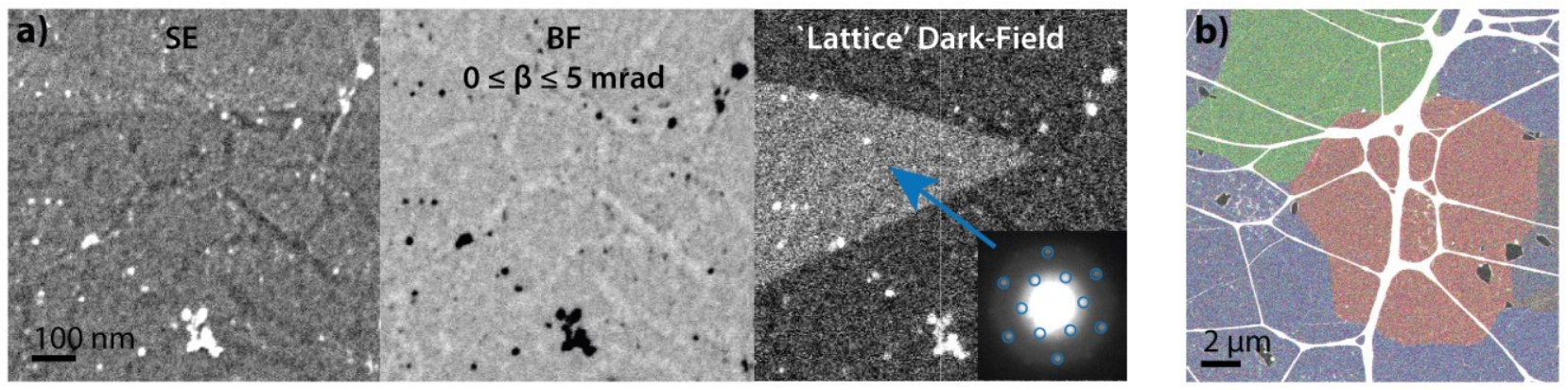

Figure 2. a) Secondary-electron, bright-field, and 'lattice' dark-field images of monolayer graphene. The 'lattice' dark field was collected with a mask on the DMD corresponding to the indicated regions of the diffraction pattern in the inset. b) An (RGB) color-composite image of three different 'lattice' dark field images over a large field of view (ca. $19 \mu \mathrm{m})$. Individual grains of graphene have color contrast, while the support film is white. 